\title{
Identification of Cacao TIR-NBS-LRR Resistance Gene Homologues and Their Use as Genetic Markers
}

\author{
David N. Kuhn ${ }^{1}$ and Giri Narasimhan ${ }^{2}$ \\ Department of Biological Sciences, Florida International University, University Park Campus, Miami, \\ FL 33199 \\ Kyoko Nakamura, J. Steven Brown, Raymond J. Schnell, and Alan W. Meerow \\ USDA-ARS, Subtropical Horticulture Research Station, 13601 Old Cutler Rd., Miami, FL 33158
}

\begin{abstract}
AdDitional INDEX words. Theobroma cacao, capillary array electrophoresis (CAE), single-strand conformational polymorphism (SSCP), single-nucleotide polymorphism (SNP), candidate genes, non-TIR-NBS-LRR resistance gene homologues, high-throughput genetic analysis

Aвstract. Identifying genetic markers linked to disease resistance in plants is an important goal in marker-assisted selection. Using a candidate-gene approach, we have previously developed genetic markers in cacao (Theobroma cacao L.) for two families of genes involved in disease resistance: non-TIR-NBS-LRR (Toll/Interleukin-1 Receptor-nucleotide binding site-leucine rich repeat) resistance gene homologues and WRKY transcription factor genes; however, we failed to isolate TIR-NBS-LRR genes. Using a novel algorithm to design degenerate primers, we have now isolated TIR-NBS-LRR loci as determined by DNA sequence comparison. These loci have been developed as genetic markers using capillary array electrophoresis (CAE) and single-strand conformational polymorphism (SSCP) analysis. We have mapped three distinct TIR-NBS-LRR loci in an $F_{2}$ population of cacao and demonstrated that one is located on linkage group 3 and the other two on linkage group 5.
\end{abstract}

The NBS-LRR (nucleotide binding site-leucine rich repeat) resistance gene family produces gene products that play an early role in signal transduction and the identification of pathogens for disease resistance (Hulbert et al., 2001). In Arabidopsis thaliana (L.) Heynh., 149 NBS-LRR genes have been identified (Meyers et al., 2003). Although few of these genes have as yet been correlated with resistance to a specific pathogen, they comprise an important group of genes that are the most likely candidates to be developed as markers for disease resistance. The TIR (Toll/ Interleukin-1 Receptor) motif is found in approximately $50 \%$ of the NBS-LRR resistance genes or putative resistance genes in A. thaliana characterized to date and is not present in resistance genes in monocots (Hulbert et al., 2001). We previously took a candidate-gene approach to identifying genes associated with disease resistance in Theobroma cacao (Borrone et al., 2004; Kuhn et al., 2003). The candidate genes identified were converted into molecular markers by single-strand conformational polymorphism (SSCP) analysis (Kuhn and Schnell, 2005; Kuhn et al., 2005). Although we previously identified 10 categories of resistance gene homologues (RGH) in cacao, none of them contained the TIR motif. One of the RGH loci mapped close to a quantitative trait locus (QTL) for witches' broom resistance (Brown et al., 2005). In the hope of finding more closely associated genetic markers, we attempted to isolate TIR-containing resistance gene homologues from T. cacao. We employed a new algorithm for the design of degenerate primers (Wei et al., 2003) and focused

Received for publication 7 Dec. 2005. Accepted for publication 23 June 2006. We thank Masterfoods, Inc., and their funding through the Cooperative Research and Development Agreement (CRADA) to the United States Department of Agriculture-Agricultural Research Service, Subtropical Horticulture Research Station (USDA-ARS SHRS) Cacao Program, that supported in part this research. 1To whom reprint requests should be addressed; e-mail: kuhnd@fiu.edu ${ }^{2}$ School of Computing and Information Science, Florida International University, University Park Campus, Miami, FL 33199. on cloning TIR-NBS-LRR from 'TSH516', the $\mathrm{F}_{1}$ individual that was used to generate the $\mathrm{F}_{2}$ population in which we have mapped our other candidate genes (Brown et al., 2005). We report here the identification and mapping of three loci of TIR-NBS-LRR genes from T. cacao.

\section{Materials and Methods}

Plant materials. Theobroma cacao clone 'TSH516' was used as the source of DNA for amplification with degenerate primers. 'TSH516' was selfed to create the $\mathrm{F}_{2}$ population (Brown et al., 2005) that is segregating for resistance to witches' broom [Moniliophthora perniciosa (Stahel) Aime and Phillips-Mora]. The $146 \mathrm{~F}_{2}$ plants derived from 'TSH516' were used to establish the map locations of the TIR-NBS-LRR loci.

Degenerate PRIMER Design. Degenerate primers to TIRNBS-LRR genes were designed as described in Wei et al. (2003) using the DePiCt program (Wei and Narasimhan, 2005). Briefly, 47 full-length amino acid sequences of TIR-NBS-LRR genes from different plants were aligned using PILEUP (Accelrys, Carlsbad, Calif.). For the accession numbers of the sequences, please refer to Wei et al., 2003, a copy of which can be found on the DePiCt website (Wei and Narasimhan, 2005). The DePiCt program found regions of identity among the aligned sequences and reverse translated them into degenerate primer sequences. If the degeneracy of any primer was above 1200 -fold, the program split the alignment into subgroups and searched again for regions of identity that would reverse translate into primers of degeneracy less than 1200 -fold. This process resulted in eight pairs of primers which were designed (Table 1) to six different subgroups from the original alignment. Primers were produced by Sigma-Genosys (The Woodlands, Texas).

DNA ISOLATION AND PCR AMPLIFICATION. DNA extraction and amplification with degenerate primers were performed as described 
Table 1. Degenerate primers designed for amplification of Toll/Interleukin-1 Receptor-nucleotide binding site-leucine rich repeat (TIR-NBS-LRR) resistance gene homologues in Theobroma cacao.

\begin{tabular}{|c|c|c|c|c|}
\hline $\begin{array}{l}\text { Group } \\
\text { no. }\end{array}$ & Degenerate primers $^{\mathrm{z}}$ & $\begin{array}{l}\text { Degeneracy } \\
\text { (fold) }\end{array}$ & $\begin{array}{c}\text { Primer } \\
\text { location }\end{array}$ & $\begin{array}{c}\text { Primer amino acid } \\
\text { sequence }^{\mathrm{x}}\end{array}$ \\
\hline \multirow[t]{3}{*}{1} & F1-GGNCCNGGRWSTCGKATTATDATCAC & 768 & $530-538$ & GPGSRIIIT \\
\hline & F2-GAYGCNYTTCARATHTTYTG & 192 & $566-572$ & EA[FL]QIFC \\
\hline & R-DRTYTCYCKNCCVARYTG & 1152 & $746-741$ & Q[FL]GRE[TI] \\
\hline \multirow[t]{2}{*}{2} & F-ATNRTHGGNATHTGGGGN & 1152 & $403-408$ & {$[\mathrm{MI}][\mathrm{IV}] \mathrm{GIWG}$} \\
\hline & R-RTCNGGDAKYTCYTTYAR & 768 & 937-932 & $\mathrm{LKE}[\mathrm{LI}] \mathrm{PD}$ \\
\hline \multirow[t]{2}{*}{3} & F-GGNATHGGNAARACNAC & 384 & $411-416$ & GIGKTT \\
\hline & R-AARCANGCHATRTSVARRAA & 1152 & $664-658$ & FL[HD]IACF \\
\hline \multirow[t]{3}{*}{4} & F-GGNAARACNACNMTHGC & 768 & $413-418$ & GKTT[IL]A \\
\hline & R1-RCANGCDATRTCRAGRAA & 192 & & \\
\hline & R2-RCANGCDATRTCYARRAA & 384 & $663-658$ & FLDIAC \\
\hline \multirow[t]{4}{*}{5} & F1-SARAAYTAYGCNTCNTCN & 1024 & $245-250$ & [EQ]NYASS \\
\hline & F2-SARAAYTAYGCNAGYTCN & 512 & & \\
\hline & R1-TCYCTNGTNGTDATDATDA & 576 & $541-535$ & [VI]IITTRD \\
\hline & R2-TCNCGNGTNGTDATDATDA & 864 & & \\
\hline \multirow[t]{2}{*}{6} & F-TTYAGNGGNGARGAYGTN & 512 & $187-192$ & FRGEDV \\
\hline & R-GCDATNGTNGTYTTNCC & 384 & $418-413$ & GKTTIA \\
\hline
\end{tabular}

2International Union of Pure and Applied Chemistry (IUPAC) nucleotide degeneracy code is $\mathrm{M}$ [A or $\mathrm{C}], \mathrm{R}$ [A or G], W [A or T], $\mathrm{S}$ [C or G], Y

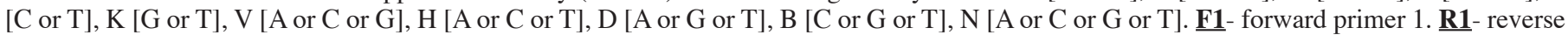
primer 1 , etc.

yIndicates position of primer in the aligned amino acid sequences described in Wei et al. (2003).

Brackets indicate degeneracy in amino acid sequence \{e.g., [FL] indicates F or L at this position\}.

in Kuhn et al. (2003), with the exception that Platinum Taq High Fidelity (Invitrogen, Carlsbad, Calif.) was used. A temperature gradient $\left(45\right.$ to $\left.65^{\circ} \mathrm{C}\right)$ was performed on the PTC-0225 DNA Engine Tetrad (MJ Research, Inc., Waltham, Mass.) to determine the optimum temperature for amplification of the eight sets of TIR-NBS-LRR degenerate primers.

Cloning ANd SEQuencing of PCR PRoducts. Amplification products were electrophoresed on a $1.5 \%$ agarose gel in $0.5 \mathrm{x}$ TBE and stained with ethidium bromide. Fragments larger than $500 \mathrm{bp}$ were excised from the gel, extracted with a Qiaquick Gel Extraction Kit (Qiagen, Valencia, Calif.) and cloned into the Topo4 vector (Invitrogen) according to the manufacturer's specifications. Clones were selected by growth in the presence of ampicillin (Borrone et al., 2004). Individual clones were amplified with M13 primers (Kuhn et al., 2003) and the amplified fragments sequenced on an ABI3100 (Applied Biosystems Inc., Foster City, Calif. ) using either T3 or T7 as the sequencing primers (Borrone et al., 2004).

Sequence analysis. Unedited sequence data were analyzed by several methods using programs from GCGDNA analysis software (Accelrys Software Inc., San Diego). The unedited sequences were made into a dataset and searched using TFASTX. The query sequences were either full-length gene sequences or partial sequences that represent the expected amplified regions from the subgroup sequences used to design the degenerate primers (Wei et al., 2003). In addition, all 460 unedited nucleotide sequences were used to query plant sequences in the GenBank database in a BLASTN (basic local alignment search tool-nucleotide) and a TBLASTX (translated basic local alignment search tool) search. The searches were done after $2000 \mathrm{hr}$ EST using the blastcl3 executable program [National Center for Biotechnology Information (NCBI), 2004] and all 460 sequences were in a single search file in FASTA format. The single output BLAST report for all 460 sequences was parsed using a shell script (available on request) to generate a spreadsheet file containing the top three hits for each query sequence.
Sequences identified by BLAST report to have significant identity to known TIR-NBS-LRR genes (E value $<\mathrm{e}^{-35}$, where the $E$ value is the number of alignments that would be expected by chance alone) were edited for both vector and degenerate primer sequences and aligned using the PILEUP program of GCG. Pairwise distances were calculated for both nucleotide and amino acid sequences using the OLDDISTANCES program of GCG. $\mathrm{K}_{\mathrm{a}}$ and $\mathrm{K}_{\mathrm{s}}$ values for the aligned nucleotide sequences were calculated using the DIVERGE program of GCG. Clustering of TIR-NBSLRR sequences was performed using the unweighted pair group method, arithmetic mean (UPGMA) algorithm, and phylograms prepared by the GROWTREES program of GCG.

DESIGN OF SSCP PRIMERS. The aligned edited nucleotide sequences were used to design primers using the PRIME+ program of GCG for individual loci (TIR2-4). Regions were chosen that included a single-nucleotide polymorphism (SNP) and primers were designed in regions that flanked the polymorphism and generated a fragment between 150 and 250 nucleotides (nt) to maximize efficiency of detection by SSCP analysis. Forward primers were labeled with 6FAM (6-carboxyfluorescein) at the 5 ' end and reverse primers were labeled with HEX (6-carboxy$2^{\prime}, 4,4^{\prime}, 5^{\prime}, 7,7^{\prime}$-hexachlorofluorescein ) at the 5' end to enable detection of each strand under nondenaturing conditions. Primers were synthesized by Sigma-Genosys.

PCR AND SSCP ANALYSIs. DNA from the 146 trees of the $\mathrm{F}_{2}$ population was amplified with the SSCP primers specific for TIR2, 3, and 4 as described in Kuhn et al. (2003). SSCP analysis was performed as described in Kuhn and Schnell (2005) with the following modifications. Amplified fragments were diluted 1:100 with sterile distilled $\mathrm{H}_{2} \mathrm{O}$ and $10 \mu \mathrm{L}$ of sample was denatured for 5 min at $95^{\circ} \mathrm{C}$ and snap cooled on ice. Ten microliters of 1:200 diluted undenatured ROX2500 molecular weight standards (Applied Biosystems Inc.) were added to each sample; the samples were then analyzed on the ABI3100 (Applied Biosystems Inc.) using a 50-cm capillary array with $5 \%$ GeneScan polymer, $10 \%$ glycerol, 1xTBE at 22 and $28{ }^{\circ} \mathrm{C}$. Run files were analyzed with 
GeneScan and Genotyper software (Applied Biosystems Inc.) and genotypes exported as tables for mapping.

MAPPING OF TIR-NBS-LRR LOCI. Mapping of TIR-NBS-LRR loci was as described in Brown et al. (2005). The TIR-NBSLRR loci were mapped together with 192 SSR markers and 10 candidate-gene markers in the $\mathrm{F}_{2}$ population, providing a solid mapping background for their placement.

\section{Results}

Amplification of $T$. CACAO Genomic DNA With degenerate PRIMERS FOR TIR-NBS-LRR GENES. Genomic DNA of 'TSH516', the parent of the $\mathrm{F}_{2}$ mapping population, was amplified with the six different groups of primers described in Table 1 using an annealing temperature gradient from 45 to $65^{\circ} \mathrm{C}$. Groups 2 and 5 primer amplification products were smears with no bands of the expected size when analyzed by agarose gel electrophoresis (data not shown). Groups 1, 3, 4, and 6 produced amplicons of the expected size and larger (see size range in Table 2) at all temperatures used in the gradient. Because the primers for group 3 and 4 were almost identical, fragments of the expected size ( 750 nt) from group 3 and 4 amplifications at all temperatures of the gradient were excised and pooled and the same was done for larger fragments (>750 nt) prior to cloning (Table 2). Group 1 and 6 fragments greater than $500 \mathrm{nt}$ were excised and cloned.

SEQUENCE ANALYSIS. The amino acid sequences of the expected fragments from known TIR-NBS-LRR were used to screen the nucleotide sequence data using TFASTX. None of the clones tested for Primer Group 1 or Primer Group 6 were TIR-NBSLRR fragments (Table 2). Fifty-one of the 110 clones (46\%) from amplicons of the expected size ( $750 \mathrm{nt})$ from Primer Group 3 and 4 showed significant identity with TIR-NBS-LRR sequences. None of the larger fragments from Primer Group 3 and 4 were identified as TIR-NBS-LRR sequences (Table 2).

All 460 sequences were used as queries against the NCBI nr database in a BLASTN and a TBLASTX search. Primer Group 1 sequences had significant identity with an $\mathrm{N}$-acetyltransferase (NM_128564.1) and a protein kinase (NM_122306.2). The sequence with the lowest expectation score $\left(2.00 \mathrm{e}^{-83}\right)$ was a UDP-glucose dehydrogenase from Populus tomentosa Carriere (AY466400.1). The T. cacao fragment matching this sequence has forward primer 1 (Group 1 F1 Table 2) at both the 5' and 3' ends of the sequence. Similarly, the T. cacao fragments matching the protein kinase sequences had forward primer 2 (Group 1 F2 Table 2) at both the 5' and 3' ends. For other T. cacao Group 1 sequences that showed significant identity with plant sequences other than TIR-NBS-LRR, the reverse primer was found at the 5 ' end of the amino acid sequence and the forward primer at the 3 ' end. Similar problems were found with the Group 6 sequences which also had significant matches with NCBI sequences but where the reverse primer was found at both the 5' end and the 3 ' end of the sequence.

The 51 clones from Group 3 and 4 that matched TIR-NBS-LRR sequences were sequenced from both the 5' and 3' direction and the combined sequences aligned. Primer regions and duplicate identical sequences were removed from the aligned sequences and the sequences were realigned. The 30 realigned sequences fell into nine well-separated clusters (Fig. 1). When the sequences were translated, clusters 4B, 7B, 8, and 9 contained single-nucleotide deletions in all members that caused frameshifts and early termination of the protein. As NBS-LRR genes are frequently found as pseudogenes, these clusters were not considered for specific primer generation. Clusters 1A, 2, 3, and 4A were chosen for specific primer generation because each appeared to represent separate alleles of a single heterozygous locus that could be mapped in the $\mathrm{F}_{2}$ population. Clusters $1 \mathrm{~B}, 5$, and 6 all had more than two closely related sequences, which might suggest a duplicated locus. Cluster 7 had only three sequences but they were all identical, which would not allow mapping in the $\mathrm{F}_{2}$ population.

A single representative sequence with a continuous open reading frame from clusters 1A, 2, 3, 4A, 5, 6, and 7 and two sequences from cluster $1 \mathrm{~B}$ were aligned and translated. The pairwise uncorrected distance of the nucleotide sequences and the translated amino acid sequences was calculated (Table 3 ). Pairwise identity between nucleotide sequences was always greater than between amino acid sequences, an unexpected result. The rate of synonymous substitution at synonymous sites $\left(\mathrm{K}_{\mathrm{s}}\right)$ and nonsynonymous substitution at nonsynonymous sites $\left(\mathrm{K}_{\mathrm{a}}\right)$ was calculated using the DIVERGE program of GCG (data not shown). The $\mathrm{K}_{\mathrm{a}} / \mathrm{K}_{\mathrm{s}}$ ratio for any pair of sequences was not greater than 1; thus positive selection in this region is not supported.

The TIR-NBS-LRR genes of $A$. thaliana have all been identified and placed in groups by sequence alignment (Meyers et al., 2003). The $A$. thaliana amino acid sequences were trimmed and

Table 2. Results of amplification of Theobroma cacao genomic DNA with degenerate primers designed to Toll/Interleukin-1 Receptor-nucleotide binding site-leucine rich repeat (TIR-NBS-LRR) resistance genes.

\begin{tabular}{|c|c|c|c|c|c|}
\hline \multicolumn{2}{|c|}{ Primer combination } & \multirow{2}{*}{$\begin{array}{c}\begin{array}{c}\text { Expected product size } \\
(\mathrm{nt})^{\mathrm{z}}\end{array} \\
648\end{array}$} & \multirow{2}{*}{$\frac{\text { Amplified product }}{\text { multiple bands }(400-900 \mathrm{nt})}$} & \multirow{2}{*}{$\begin{array}{c}\begin{array}{c}\text { Clones sequenced } \\
\text { (no.) }\end{array} \\
96\end{array}$} & \multirow{2}{*}{$\begin{array}{c}\text { TIR-NBS-LRR } \\
\text { sequences identified } \\
\text { (no.) } \\
0\end{array}$} \\
\hline$\overline{\mathrm{G} 1 \mathrm{a}}$ & 1F1-1R & & & & \\
\hline G1b & $1 \mathrm{~F} 2-1 \mathrm{R}$ & 540 & smear & 0 & 0 \\
\hline G2 & $2 \mathrm{~F}-2 \mathrm{R}$ & 1602 & smear & 0 & 0 \\
\hline G3 & $3 F-3 R$ & 759 & three bands $(750,1300,2000 \mathrm{nt})$ & $(\sim 750 \mathrm{nt}) 110^{\mathrm{y}}$ & 51 \\
\hline G4a & $4 \mathrm{~F}-4 \mathrm{R} 1$ & 750 & two bands $(750,2000 \mathrm{nt})$ & $(>750 \mathrm{nt}) 175^{\mathrm{x}}$ & 0 \\
\hline G4b & $4 \mathrm{~F}-4 \mathrm{R} 2$ & 750 & none & 0 & 0 \\
\hline G5a & $5 F 1-5 R 1$ & 888 & none & 0 & 0 \\
\hline G5b & $5 F 1-5 R 2$ & 888 & none & 0 & 0 \\
\hline G5c & $5 F 2-5 R 1$ & 888 & none & 0 & 0 \\
\hline G5d & $5 F 2-5 R 2$ & 888 & none & 0 & 0 \\
\hline G6 & $6 \mathrm{~F}-6 \mathrm{R}$ & 693 & multiple bands (400-900 nt) & 79 & 0 \\
\hline
\end{tabular}

znt = nucleotides.

yAmplicons from primer combination G3 and G4a of $~ 750 \mathrm{nt}$.

xAmplicons from primer combination G3 and G4a of $>750 \mathrm{nt}$. 


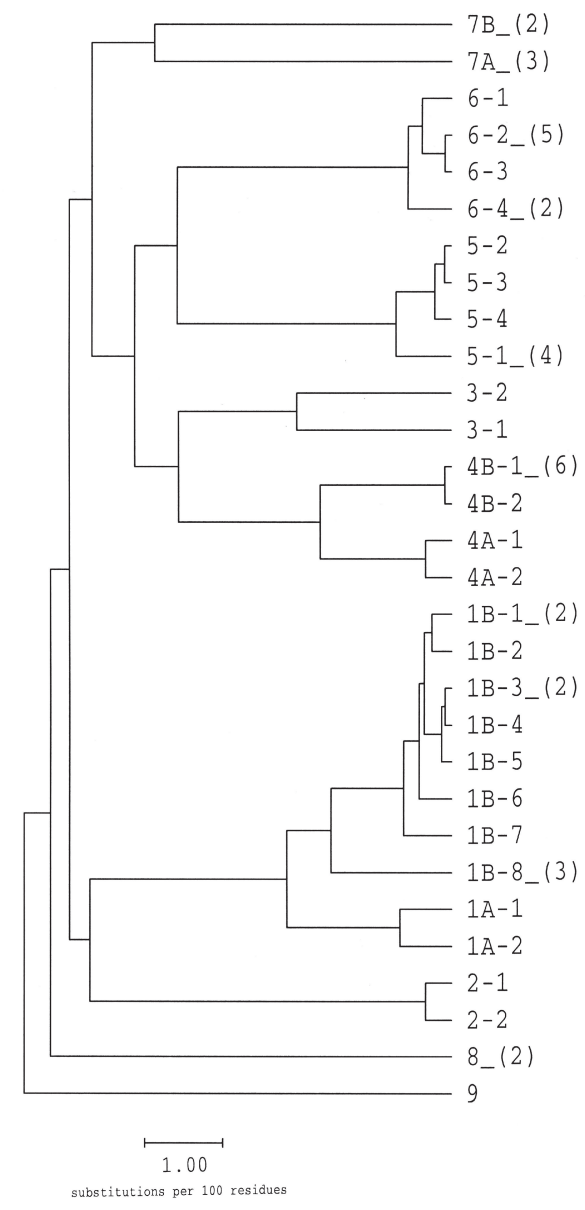

Fig. 1. A dendrogram of the 51 sequences identified as Toll/Interleukin-1 Receptor-nucleotide binding site-leucine rich repeat (TIR-NBS-LRR) from Theobroma cacao. Cluster and sequence designators are at the ends of the branches (e.g., 1A-1 is cluster 1A sequence 1). Clusters are not in numerical order because they were defined by sequence alignment prior to creation of the dendrogram. Numbers in parentheses after the cluster and sequence designator denote the number of identical sequences found. The length of the branches is proportional to the uncorrected distance between the aligned nucleotide sequences. The bar represents the length equal to 1.00 nucleotide substitution per 100 residues. aligned with the translated T. cacao sequences. Adendrogram was generated by UPGMA using the uncorrected pairwise distances from the aligned sequences (Fig. 2). Theobroma cacao sequences are most closely related to TNL-C (TIR-NBS-LRR Group C) sequences from A. thaliana (Fig. 2). The T. cacao TIR-NBS-LRR fragments were aligned with a single representative of $A$. thaliana group TNL-C (At1g27180) (Fig. 3). The VLLVLDDVD motif of the kinase 2 region, which is diagnostic for TIR-NBS-LRR sequences, is highlighted. The other diagnostic sequence for TIRNBS-LRR was the site for the reverse primer for Primer Group 3 and 4 and was trimmed from the T. cacao sequences prior to alignment. All the T. cacao TIR-NBS-LRR sequences identified in this study contained the amino acid motif MFDLEASSSYGDLCKV (highlighted in Fig. 3).

MAPPING. Because the sequences were more than $90 \%$ identical at the nucleotide level, it was possible that all sequences were from a single cluster of duplicated genes on a single chromosome. Primers were designed from the aligned sequences to discriminate between clusters and to incorporate potential SNPS (Fig. 4). Amplification of genomic DNA with primers designed to Cluster 1A sequences produced fragments representing more than two alleles and could not be used for mapping (data not shown). Primer pairs designed to flank SNPs in TIR2, TIR3, and TIR4 (Table 4) were used to map these loci in the $\mathrm{F}_{2}$ population. Although the sequences shared a great deal of identity, the TIR2 locus was in linkage group 1 while TIR 3 and TIR4 were in linkage group 5 (Fig. 5). TIR3 and TIR4 are about $0.3 \mathrm{cM}$ apart. In cacao, a centiMorgan (cM) is approximately $330 \mathrm{~kb}$ (Lanaud et al., 2004), thus making TIR3 and TIR4 about $100 \mathrm{~kb}$ apart. TIR2, TIR3 and TIR4 did not map close to either the major or minor QTL for witches' broom resistance (Fig. 5).

\section{Discussion}

In dicots, the family of resistance genes is divided into two main groups, those with a Toll/Interleukin-1 Receptor motif (TIR) and those without (non-TIR). Several groups have found it difficult with degenerate primers designed to the highly conserved portions of the NBS region to isolate both non-TIR and TIR-NBS-LRR containing resistance gene homologues (Kuhn et al., 2003; Noir et al., 2001; Tian et al., 2004). Using a novel approach to the designing of degenerate primers (Wei et al., 2003),

Table 3. Pairwise comparison of uncorrected nucleotide and amino acid distances for representative sequences from Toll/Interleukin-1 Receptornucleotide binding site-leucine rich repeat (TIR-NBS-LRR) sequence clusters of Theobroma cacao. Clusters as described in Fig. 1 are not in numerical order because they were defined by sequence alignment prior to generation of the distance table.

\begin{tabular}{|c|c|c|c|c|c|c|c|c|c|}
\hline \multirow{3}{*}{$\begin{array}{l}\text { Nucleotide } \\
\text { distances }\end{array}$} & \multicolumn{9}{|c|}{ Amino acid distances } \\
\hline & \multicolumn{9}{|c|}{ Sequence cluster } \\
\hline & $1 \mathrm{~A}$ & 1B-1 & $1 \mathrm{~B}-2$ & 2 & 3 & 6 & 5 & $4 \mathrm{~A}$ & 7 \\
\hline $1 \mathrm{~A}$ & 1 & 0.9224 & 0.9353 & 0.8448 & 0.8578 & 0.8276 & 0.8405 & 0.8448 & 0.8448 \\
\hline 1B-1 & 0.9569 & 1 & 0.9526 & 0.8147 & 0.8664 & 0.8534 & 0.8578 & 0.8578 & 0.8621 \\
\hline 1B-2 & 0.9612 & 0.9655 & 1 & 0.8448 & 0.8621 & 0.8276 & 0.8448 & 0.8448 & 0.8405 \\
\hline 2 & 0.9109 & 0.8951 & 0.9124 & 1 & 0.8664 & 0.8362 & 0.8405 & 0.8578 & 0.8405 \\
\hline 3 & 0.9066 & 0.9095 & 0.8966 & 0.9037 & 1 & 0.8836 & 0.8793 & 0.9052 & 0.8922 \\
\hline 6 & 0.8994 & 0.8966 & 0.8851 & 0.8937 & 0.9167 & 1 & 0.8793 & 0.8922 & 0.8879 \\
\hline 5 & 0.908 & 0.9109 & 0.898 & 0.9124 & 0.9195 & 0.9253 & 1 & 0.8836 & 0.8793 \\
\hline $4 \mathrm{~A}$ & 0.9124 & 0.9095 & 0.9066 & 0.9152 & 0.9253 & 0.9195 & 0.9267 & 1 & 0.9052 \\
\hline 7 & 0.9037 & 0.8994 & 0.8951 & 0.9037 & 0.908 & 0.908 & 0.9109 & 0.9138 & 1 \\
\hline
\end{tabular}




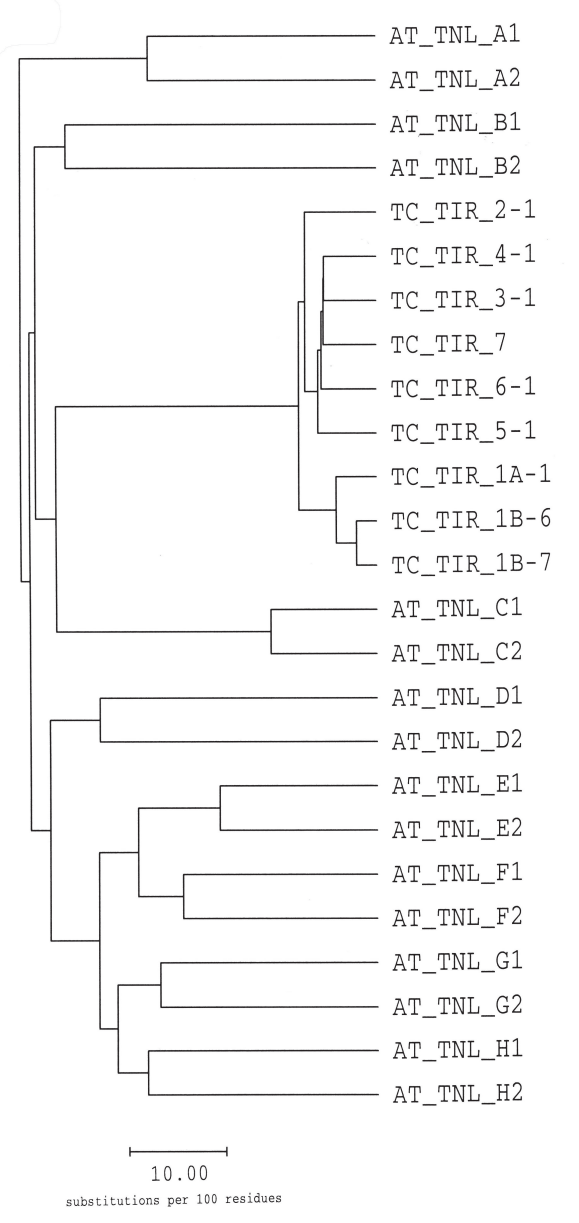

Fig. 2. A dendrogram of representative sequences from the seven Theobroma cacao clusters that contained complete reading frames with representative sequences from eight groups (A-H) of Toll/Interleukin-1 Receptor-nucleotide binding site-leucine rich repeat (TNL) sequences from Arabidopsis thaliana (At). Cluster and sequence designators are at the ends of the branches (e.g., Tc_TIR_1A-1 is $T$. cacao cluster 1A sequence 1, At_TNL_A1 is A. thaliana cluster A sequence 1).The branch lengths are proportional to the uncorrected distance between the aligned amino acid sequences. The bar represents 10 substitutions per 100 residues. The Genbank accession numbers of the At_TNL sequences used are as follows: A1-At3g51560, A2-At2g17050, B1-At4g12010, B2-At3g51570,C1-At1g27180,C2-At1g27170,D1-At4g09430,D2-At4g09360, E1-At4g16920,E2-At4g16900, F1-At4g08450,F2-At1g31540, G1-At3g44630, G2-At1g69550, H1-At1g56540, H2-At2g16870. we created six sets of degenerate primers that were targeted to TIR-NBS-LRR sequences.

All primer groups were tested against 'TSH516', the $\mathrm{F}_{1}$ individual that is the parent of the only extant $\mathrm{F}_{2}$ population of $T$. cacao. This $\mathrm{F}_{2}$ population has proved useful as a mapping population for both microsatellite and SSCP-based markers (Brown et al., 2005). Two of the primer groups that targeted the same regions were the only groups successful in amplifying TIR-NBSLRR sequences. These sequences were identified by comparison with known TIR-NBS-LRR sequences and the presence of the diagnostic amino acid sequence in the kinase 2 region (VLLVLDDVD) which was found in all of the TIR-NBS-LRR sequences in T. cacao while VLLVLDDVW was found in all the non-TIRNBS-LRR sequences previously identified in T. cacao (Kuhn et al., 2003). When the TIR-NBS-LRR sequences were aligned with the non-TIR-NBS-LRR sequences from cacao, they formed two distinct clusters with no overlap (data not shown). Thus, for both the T. cacao non-TIR and TIR-NBS-LRR sequences, they are more similar to non-TIR or TIR-NBS-LRR sequences from other species than they are to each other.

Two of the primer groups ( 2 and 5 ) failed to amplify distinct fragments in T. cacao, which may be due to the great number of potential annealing sites for these degenerate primers in the cacao genome. When primer groups 2 and 5 were tested for amplification with an annealing temperature gradient, no specific fragments were amplified and no further amplifications were attempted. However, the forward primers from groups 2 and 5 could be paired with the reverse primers from sets 3 and 6 , respectively, to determine if TIR-NBS-LRR specific fragments can be amplified.

Primer groups 1 and 6 gave distinct amplification products in the size range expected, but none of these products were related to TIR-NBS-LRR genes. Analysis of the sequences that were identified by BLAST searches of the NCBI database showed that the most frequent products amplified were often due to annealing of a single primer, such as the Group 1 forward primer F1 or F2, at both the 5' and 3' ends of the amplified fragment. Such unintended amplicons are frequently encountered when using degenerate primers. This would also suggest that use of the Group 1 forward primers with other reverse primers might lead to the same results.

Distances between the TIR-NBS-LRR clades were much smaller than those for the non-TIR-NBS-LRR clades previously identified. Using our previous criterion, less than $80 \%$ unweighted nucleotide distance, for dividing non-TIR-NBS-LRR sequences

1

kinase 2

120

TC_TIR_2-1 KvVYNLNIQRFESS.SFLANVRKiTQEhNGLIRLQRQLISDILKGKANKIYNSNDGI TKIKEVISRRRVLFVLDDVDDSEKITKIIEVQIPFHPGSKIIITSRqGCLLSDPFIRQMFDLE TC_TIR 1A-1 KIVYNqNfQRFESY. SFLADVRE tTQE SNGLVqLQkQLISDILKGKANKIYNIDDGI tKIKE tIChRRVLLVLDDVDDSkKVTeIIGVQIPFHPGSKIIVTSRHICLLSDIFIKQMFDLE TC_TIR_4-1 KIVYNLNIQRFESF. SFLAgVRdi iQERNGLVRLQRQLISDILKGKANKIYNSDDGI IKIKEAVIRRRVLLVLDDVDDSdiITKIIGAQIPFHPGSKIIITSRHQCLLSDPFIRQMFDLE TC TIR 3-1 KvVYNLNIQRFESF. SFLADVRE tTQERNGLVhLQRQLISDILKGKANKIYNSDDGI IKIKEAIHRERVLLVLDDVDDSEKIT IIIGaKIPFHPGSKIIITSRHICLLSDPFIRQMFDLE

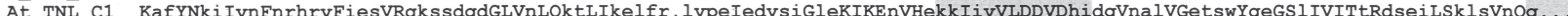
Consensus K-VYNLNIQRFESF-SFLADVRE-TQERNGLVRLQRQLISDILKGKANKIYNSDDGI-KIKEAIHRRRVLLVLDDVDDS-KIT-IIG-QIPFHPGSKIIITSRH-CLLSDPFIRQMFDLE 121

TC_TIR_2-1 ASSSYqDLCKVFEVKELLFNESLQLFKWYAFR. QNDPIDSYMEYAISIVKHCGGLPLALQVLGSSL. AGKSINVWKSALEKLKTIPDSKIQKILKISYDYLQDDHDK TC TIR $\overline{1 A}-1$ ASSNYGDLCKVFEVKE LAFNESLQLFNWYAFR. QNDPI SYMdYAKSIVKHCGGLPLALQVLGSSL. SSKSINVWISALEKLKAIPhSKIQKILRISYgSLQDDHDK TC_TIR_4-1 ASSSYGDLCKVFEVKELAFNESLQLFNWYAFg . QNDPIDSY IEYAYSVVKHCGGLPLALQVLGSSL. SGKSINVWrSALEKLqAIPDSKIQKILRISYDYLQDDHDK TC TIR 3-1 ASSShGDLCKVFEVKELAFNE SLQLFDWYAFG. QNDPID SYMEYAKS IVKHCdGLPLALQVLGSSL . GGKS INVWKSALEKLKAIPDSKIQKILRISYDSLQDDHDK

At TNL C1 ......... YEVKcLtepqaLkLFsfYslRkekpPtqgllelsKkIaevtGlLPLAvkVfGShfyd.KdeNeWqveLEKL. ktqqdKIhgVLalSFkSL.DeeeK Consenšs ASSSYGDLCKVFEVKELAFNESLOLFNWYAFR-ONDPIDSYMEYAKSIVKHCGGLPLALOVLGSSL--GKSINVW-SALEKLKAIPDSKIOKILRISYDSLODDHDK

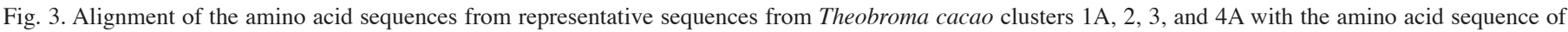

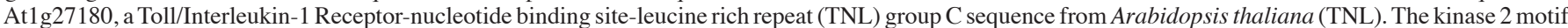

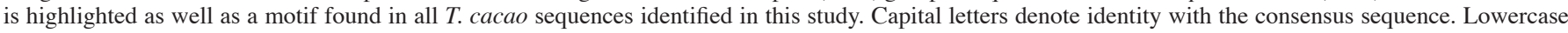
letters denote difference with the consensus sequence. Dashes in the consensus sequence represent no consensus. Dots in the aligned sequences denote gaps. 


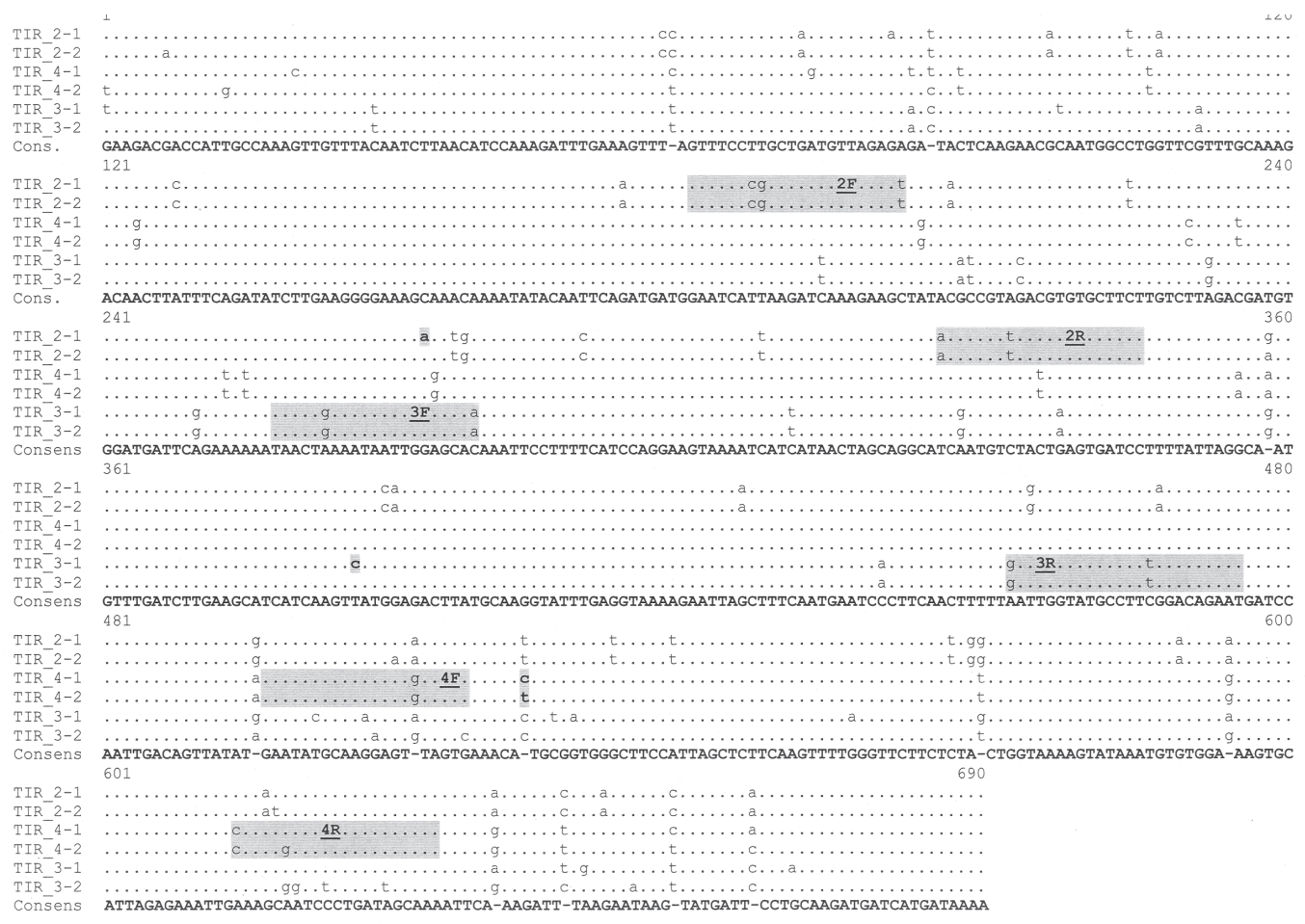

Fig. 4. A portion of the alignment of the nucleotide sequences from Theobroma cacao clusters 2, 3 and 4A. Sequence TIR_2-1 refers to sequence 1 of cluster 2 as described in Fig. 1, etc. Nucleotides that differ with the consensus sequence are shown in lower case. Positions of the primers described in Table 4 are highlighted and labeled (e.g., $\underline{\mathbf{2 F}}$ is the forward primer specific to cluster 2, $\underline{\mathbf{2}}$ is the reverse primer specific to cluster 2). Single-nucleotide polymorphisms (SNP) flanked by the primers are also highlighted.

into clades (Kuhn et al., 2003), all the TIR-NBS-LRR sequences would be included in one clade. Perhaps more surprising is that the genetic distance calculated between individual TIR-NBSLRR sequences was greater for the amino acid sequence than the nucleotide sequence. This was true both within and between clades. Thus, the few nucleotide differences between sequences almost always led to an alteration in the amino acid sequence. This unexpected difference in nucleotide and amino acid sequence distance was further investigated by measuring the rates of synonymous $\left(\mathrm{K}_{\mathrm{s}}\right)$ and nonsynonymous substitutions $\left(\mathrm{K}_{\mathrm{a}}\right)$. The $\mathrm{K}_{\mathrm{a}} / \mathrm{K}_{\mathrm{s}}$ ratios were in the 0.5-0.7 range for all pairwise comparisons, but none was above 1.0. A $\mathrm{K}_{\mathrm{a}} / \mathrm{K}_{\mathrm{s}}$ ratio greater than 1.0 would be evidence of positive selection for changes in the amino acid sequence in this region. The expectation for neutral drift would be nucleotide substitutions that cause no change in the amino acid sequence and a $\mathrm{K}_{\mathrm{a}} / \mathrm{K}_{\mathrm{s}}$ ratio of approximately 0.3 . Thus, there is no support for positive selection in this region. However, when aligning the TIR-NBS-LRR nucleotide sequences, we noted that two sequences in cluster 1B appeared to be recombinants, one between $1 \mathrm{~A}$ and 2 , and the other between $1 \mathrm{~A}$ and 3. Considering the highly conserved nature of the nucleotide binding region, it is interesting that nonsynonymous substitution and recombination are so frequently observed.

When the T. cacao TIR-NBS-LRR sequences were aligned with all the TIR-NBS-LRR sequences from A. thaliana, they clustered with the TNL-C group and were imbedded in the general TIR-NBS-LRR cluster, rather than being a separate cluster outside the $A$. thaliana sequences. This suggests that the degenerate primers targeted a particular subset of TIR-NBS-LRR sequences in T. cacao, an unexpected consequence of the creation of sequence subsets to reduce degeneracy in the primers. Thus, more TIR-NBS-LRR sequences may be isolated from cacao

Table 4. Specific primers for Theobroma cacao Toll/Interleukin-1 Receptor-nucleotide binding site-leucine rich repeat (TIRNBS-LRR) resistance gene homologue sequence clusters (2,3, and 4A) as described in Fig. 1 that flank a single-nucleotide polymorphism (SNP) in cultivar TSH516.

\begin{tabular}{|c|c|c|c|c|}
\hline $\begin{array}{l}\text { Sequence } \\
\text { cluster }\end{array}$ & Primer sequence & $\begin{array}{l}\text { Fragment } \\
\text { length }(\mathrm{nt})^{\mathrm{z}}\end{array}$ & SNPy & $\begin{array}{c}\text { Genotype } \\
\text { in 'TSH516' }\end{array}$ \\
\hline 2 & $\begin{array}{l}\underline{\mathbf{F}}^{\mathrm{x}} \text {-GGAATCACGAAGATCAAAGAAGT } \\
\underline{\mathbf{R}}^{\mathrm{x}} \text {-GGATCACTCAGTAAACATTGT }\end{array}$ & 167 & 1 & heterozygous \\
\hline 3 & $\begin{array}{l}\underline{\mathbf{F}} \text {-ATAACTGAAATAATTGGAGCAA } \\
\underline{\mathbf{R}} \text {-TGTCCAAAGGCATACCAATC }\end{array}$ & 215 & 1 & heterozygous \\
\hline $4 \mathrm{~A}$ & $\begin{array}{l}\underline{\mathbf{F}} \text {-AGAATATGCAAGGAGTGTAGTG } \\
\underline{\mathbf{R}} \text {-TTGCTATCAGGGATYGCTTG }\end{array}$ & 138 & 1 & heterozygous \\
\hline
\end{tabular}

${ }^{\mathrm{z} n \mathrm{t}}=$ nucleotides.

ySNP = single-nucleotide polymorphism.

$\underline{\mathbf{F}}$ - forward primer; $\underline{\mathbf{R}}$ - reverse primer. 


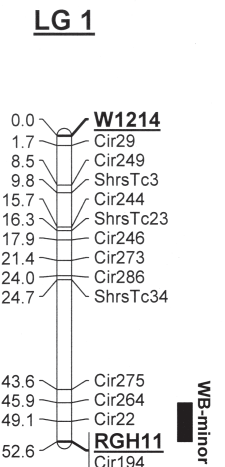

$\underline{\text { LG } 6}$

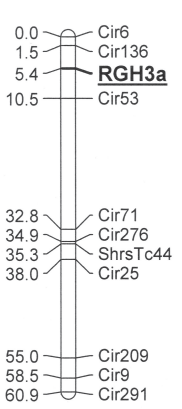

$\underline{\mathrm{LG} 2}$

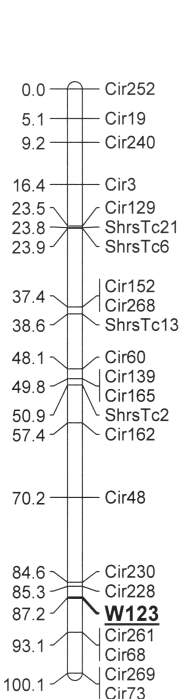

$\underline{\text { LG } 7}$

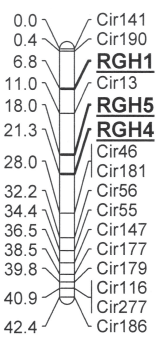

$\underline{\text { LG } 3}$

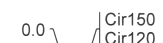

$\left.\begin{array}{l}2.1 \\ 3.8\end{array}\right] / \begin{aligned} & \text { Cir120 } \\ & \text { Cir153 } \\ & \text { Cir198 }\end{aligned}$

5.4 $)$ TIR2

9.6 Cir146

10.6 Cir21

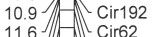

12.1 $\longrightarrow$ Cir62

$\left.\begin{array}{l}12.1 \\ 13.9\end{array}\right]\left[\begin{array}{l}C_{C i r} 40 \\ C_{C i r} 247\end{array}\right.$

16.9 $] \quad\left[\begin{array}{l}\text { Cir204 } \\ 21.5\end{array}\right.$

31.3 $7 \begin{aligned} & { }_{\text {Cir175 }} \\ & \text { Cir180 }\end{aligned}$

$39.1 \simeq$ Cir280

40.0 Cir289

41.5 Cir78

44.8 $\}{ }_{\text {Cir263 }}$

$\left.\begin{array}{l}63.6 \\ 63.9\end{array}\right]\left[\begin{array}{l}\mathrm{Cir} 254 \\ \mathrm{C} i r 135\end{array}\right.$

$\left.\begin{array}{l}63.9 \\ 66.1\end{array}\right]\left[\begin{array}{l}\text { Cir135 } \\ \text { Cir128 } \\ \text { Cir140 }\end{array}\right.$

70.0 $-\begin{aligned} & \text { Cir140 } \\ & \text { ShrsTc7 }\end{aligned}$

70.4 凋 ShrsTc5

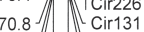

71.6 $/ \mathrm{Cir} 202$

$\left.\begin{array}{l}72.3 \\ 79.4\end{array}\right] \begin{aligned} & { }_{C}^{C i r 144} \\ & \text { Cir81 }\end{aligned}$ $\underline{\text { LG } 4}$

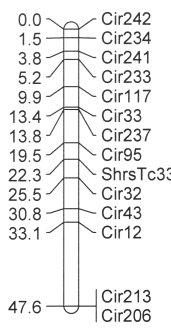

$\underline{\text { LG } 5}$

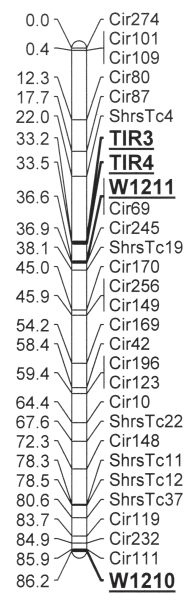

$\underline{\text { LG } 10}$

Fig. 5. A genetic linkage map of the genome of Theobroma cacao showing the position of three loci described in this paper. The linkage groups (LG1, etc.)shown are those described in Lanaud et al., 2004. The loci shown on the map are as follows: loci labeled Cir are microsatellite loci developed by Lanaud et al., 2004; loci labeled ShrsTc are microsatellite loci developed at U.S. Department of Agriculture-Agricultural Research Service, Subtropical Horticulture Research Station (USDA-ARS SHRS), Miami, Fla. (Brown et al., 2005); candidate-gene loci are shown in bold and underlined. Candidate-gene loci are as follows: loci labeled resistance gene homologue (RGHXX) are non- Toll/Interleukin-1 Receptor-nucleotide binding site-leucine rich repeat (non-TIR-NBS-LRR) resistance gene homologues (Kuhn et al., 2003); loci labeled W12XX are WRKY transcription factor genes (Borrone et al., 2004) and loci labeled TIRX are Toll/Interleukin-1 Receptor-nucleotide binding site-leucine rich repeat (TIR-NBS-LRR) resistance gene homologues described here. Quantitative trait loci (QTL) for resistance to witches' broom ( $\underline{\mathbf{W B}})$ are shown in bars next to the linkage groups (Brown et al., 2005).

by redesigning the degenerate primers to specific subgroups of the A. thaliana TIR-NBS-LRR sequences (TNL-A, etc.) as the source of the alignment for primer design. Interestingly, the $T$. cacao TIR-NBS-LRR sequences all shared a common, conserved amino acid motif (MFDLEASSSYGDLCKV) that is absent in A. thaliana sequences but which matched a similar motif in two putative TIR-NBS-LRR pseudogenes in Gossypium hirsutum L. (AY600402, AY600378; He et al., 2004), also a member of the Malvales. This motif was lacking from the other reported TIRNBS-LRR sequences from cotton (Tan et al., 2003) which were also not identified by BLAST search with the T. cacao sequences. Primers designed to this highly conserved region may prove to be the best means to isolate more genetically diverse TIR-NBSLRR sequences from cacao.
Development of molecular markers using a candidate-gene approach has proved useful and can be applied to any candidate gene. We are currently using this same method to develop markers from ESTs (expressed sequence tags) and from BAC (bacterial artificial chromosome) sequences. Because the number of expressed genes is likely in the tens of thousands for T. cacao, the method described here has the potential to generate thousands of genetic markers for placement on the cacao genetic map, many more markers than could be expected from SSRs. However, the association of such markers with interesting phenotypes requires large populations that are segregating for the phenotype of interest. In tree crops such as T. cacao, it is the lack of such populations, rather than the development of molecular markers, that is the limiting factor for advances in marker-assisted selection. 


\section{Literature Cited}

Borrone, J.W., D.N. Kuhn, and R.J. Schnell. 2004. Isolation, characterization, and development of WRKY genes as useful genetic markers in Theobroma cacao. Theor. Appl. Genet. 109:495-507.

Brown, J.S., R.J. Schnell, J.C. Motamayor, U. Lopes, D.N. Kuhn, and J.W. Borrone. 2005. Resistance gene mapping for witches' broom disease in Theobroma cacao $\mathrm{L}$. in an $\mathrm{F}_{2}$ population using SSR markers and candidate genes. J. Amer. Soc. Hort. Sci. 130:366-373.

He, L.M., C.G. Du, L. Covaleda, Z.Y. Xu, A.F. Robinson, J.Z. Yu, R.J. Kohel, and H.B. Zhang. 2004. Cloning, characterization, and evolution of the NBSLRR-encoding resistance gene analogue family in polyploid cotton (Gossypium hirsutum L.). Mol. Plant-Microbe Interactions 17:1234-1241.

Hulbert, S.H., C.A. Webb, S.M. Smith, andQ. Sun. 2001. Resistance gene complexes: evolution and utilization. Annu. Rev. Phytopathol. 39:285-312.

Kuhn, D.N., J. Borrone, A.W. Meerow, J.C. Motamayor, J.S. Brown, and R.J. Schnell. 2005. Single-strand conformation polymorphism analysis of candidate genes for reliable identification of alleles by capillary array electrophoresis. Electrophoresis 26:112-125.

Kuhn, D.N., M. Heath, R.J. Wisser, A. Meerow, J.S. Brown, U. Lopes, and R.J. Schnell. 2003. Resistance gene homologues in Theobroma cacao as useful genetic markers. Theor. Appl. Genet. 107:191-202.

Kuhn, D.N. and R.J. Schnell. 2005. Use of capillary array electrophoresis single-strand conformational polymorphism analysis to estimate genetic diversity of candidate genes in germplasm collections. Methods Enzymology 395:238-258.
Lanaud, C., A.M. Risterucci, I. Pieretti, J.A.K. N'Goran, and D. Fargeas. 2004. Characterisation and genetic mapping of resistance and defence gene analogs in cocoa (Theobroma cacao L.) Mol. Breeding 13:211-227.

Meyers, B.C., A. Kozik, A. Griego, H. Kuang, and R.W. Michelmore. 2003. Genome-wide analysis of NBS-LRR-encoding genes in Arabidopsis. Plant Cell 15:809-834.

National Center for Biotechnology Information. 2004. blastcl3 executable program. 23 May 2005. <ftp://ftp.ncbi.nih.gov/blast/executables/snapshot/2004-12-05>.

Noir, S., M.C. Combes, F. Anthony, and P. Lashermes. 2001. Origin, diversity and evolution of NBS-type disease-resistance gene homologues in coffee trees (Coffea L.). Mol. Genet. Genomics 265:654-662.

Tan, H., F.E. Callahan, X.D. Zhang, M. Karaca, S. Saha, J.N. Jenkins, R.G. Creech, and D.P. Ma. 2003. Identification of resistance gene analogs in cotton (Gossypium hirsutum L.). Euphytica 134:1-7.

Tian, Y., L. Fan, T. Thurau, C. Jung, and D. Cai. 2004. The absence of TIRtype resistance gene analogues in the sugar beet (Beta vulgaris L.) genome. J. Mol. Evol. 58:40-53.

Wei, X. and G. Narasimhan, 2005. DePiCt (v2.0) web interface. 22 Feb. 2006. <http://www.cs.fiu.edu/ giri/bioinf/DePiCt1.0/WebVersion/2depict. htm>.

Wei, X., D.N. Kuhn, and Narasimhan G. 2003. Degenerate primer design via clustering. Proc. 2003 Institute of Electrical and Electronics Engineers (IEEE) Bioinformatics Conf. p. 75-83. 